\title{
EL ECAES Y SU POSIBLE IMPACTO EN EL PEU DE LA UNIVERSIDAD SURCOLOMBIANA
}

Por:

Nelson Ernesto López Jiménez ${ }^{1}$

Doctor en Educación

Área Lenguaje y Educación

Docente Departamento de Psicopedagogía

Facultad de Educación

No hay viento favorable para quien

Universidad Surcolombiana

no sabe hacia donde se dirige

SENECA

\section{CONSIDERACIONES INICIALES}

Es necesario iniciar este artículo resaltando la importancia inusitada que las instituciones de Educación Superior le han otorgado al ECAES - Examen de Calidad de la Educación Superior,que las ha llevado incluso a determinar y orientar su dinámica académica hacia la obtención de resultados positivos en este examen, al margen o distante de sus políticas institucionales consignadas en su PEI y concretadas en los diferentes Planes de Desarrollo y de Mejoramiento Académico. Se considera importante reflexionar detenidamente sobre este aspecto porque es significativo analizar la validez de estos comportamientos en el marco de la autonomía y la integridad institucional. Pretender orientar la dinámica académica de una institución hacia la consecución de un buen resultado frente a un examen, ${ }^{2}$ es reducir de manera desconcertante y temeraria, la idea y la noción de institución universitaria. En lo que sigue intentaré referirme a la gramática básica del ECAES y del Proyecto Educativo Universitario de la Surcolombiana, para intentar en la parte final de este artículo, establecer un posible diálogo entre estas dos regulaciones. 


\section{¿QUE ES EL ECAES?}

E ECAES forma parte del Sistema de Aseguramiento de la Calidad de la Educación Superior (integrado además por el proceso de Registro Calificado, la Acreditación de Calidad y el Observatorio Laboral). Es una experiencia pedagógica y evaluativa que actualmente se encuentra en sus primeros pasos ${ }^{3}$, que no permite hablar de ella como una experiencia consolidada, es un proceso académico-evaluativo que se espera aquilatar con el correr de los años y de las experiencias formativas e investigativas que lo argumenten y sustenten.

El ECAES apunta a evaluar aquellas áreas y componentes fundamentales del saber que identifican la formación de cada profesión, disciplina u ocupación, de conformidad con los estándares de calidad, dado que se quiere velar porque todos los estudiantes que egresan de la educación superior hayan construido los conceptos y desarrollado las competencias básicas para alcanzar desempeños satisfactorios en sus actividades personales y profesionales ${ }^{4}$. Se precisa que sus objetivos están directamente orientados a:

$\checkmark$ Comprobar el grado de desarrollo de las competencias de los estudiantes que cursan el último año de los programas académicos de pregrado que ofrecen las instituciones de Educación Superior.

$\checkmark \quad$ Servir de fuente de información para la construcción de indicadores de evaluación del servicio público educativo, que fomenten la cualificación de los procesos institucionales, la formulación de políticas y faciliten el proceso de toma de decisiones en todos los órdenes y componentes del sistema educativo.

Aquí es importante resaltar que la formación de un profesional es el resultado del desarrollo de un modelo pedagógico que implica un proceso complejo constituido por un discurso instruccional o específico, estructurado a partir de tres sistemas de señales (el currículo, las prácticas pedagógicas y los criterios y procesos de evaluación), articulado o en relación a un discurso regulativo, que determina las reglas de relación y de realización de los actores del proceso formativo, es decir, los comportamientos y la naturaleza de la relaciones sociales que se establecen en el proceso de formación profesional. A partir de esta elaboración, es necesario interrogar el alcance del ECAES para abordar la complejidad del proceso formativo. Es indiscutible señalar la importancia de dicho examen como también su insuficiencia para determinar de manera taxativa y categórica la calidad de un proceso formativo ¿qué interesante resultaría hacer un seguimiento a las diferentes reacciones de las IES en relación con resultados del ECAES 2005?

Se afirma que «la información generada por el ECAES se constituye en un elemento externo, distinto y complementario a la evaluación que realiza cada institución y a las prácticas de valoración y acreditación de los programas académicos de educación superior, conducente a proporcionar insumos que generen procesos de reflexión institucional conjunta con el Estado, el sector productivo y la comunidad académica y científica» ${ }^{5}$. Vale la pena entonces interrogarnos sobre algunos aspectos 
intrínsecamente asociados a la prueba o examen, con el propósito de intentar una contextualización sólida del mismo y, a partir de ahí, ubicar en su real dimensión la experiencia ECAES.

¿Cuál es el marco teórico o conceptual que soporta o respalda el ECAES? ¿Cuáles comunidades académicas validan o respaldan dicho marco?

¿Cómo se está garantizando una coherencia entre el marco conceptual y las especificaciones o estructura de la prueba? ¿Porqué se habla de componentes de la prueba (disciplinarios) y porqué su presencia varía de manera particular dependiendo del área a evaluar?

¿ Cómo se garantiza que las diferentes preguntas que constituyen la prueba respondan al enfoque formulado en el marco teórico, es decir, cómo estar seguros que realmente son preguntas que evalúan competencias? ${ }^{6}$

¿ Cómo el procesamiento y la divulgación de los resultados obtenidos en la prueba respeta de manera seria y responsable las diferentes realidades institucionales? ¿Porqué se insiste en que el ECAES no "ranquea», si es lo que efectivamente las agencias estatales vienen propiciando a través de sus diferentes agentes? ${ }^{7}$

El ECAES es un dispositivo que responde a un proceso de evaluación externa, valdría la pena preguntarnos sobre la naturaleza de esa externalidad. Es externa porque la realiza una agencia que poco o nada tiene que ver con el proceso formativo que se realiza en la educación superior8; es externa porque es ajena a lo que realizan las instituciones y porque quienes la confeccionan son "funcionarios» que no viven la realidad académica de manera directa; es externa porque no es vista como una acción académica sino como una fría clasificación y exclusión. Estos interrogantes no pueden ser intrascendentes a la vida de las IES, razón por la cual, intento poner en el tapete de la discusión y la denuncia pública el "comportamiento ingenuo» de algunas IES, que lanzan juicios y valoraciones sobre su calidad y la de sus programas y egresados, a partir del resultado de una prueba (inclusiónexclusión) que no puede arrogarse la seriedad y complejidad de un proceso formativo en el campo profesional.

Con la intención de avanzar en una mirada argumentada al fenómeno ECAES, interesante relacionarlo con los Proyectos Educativos Universitarios conocidos como PEI, para detectar posibles vínculos o interacciones entre ellos- pero también para ubicar en su real dimensión el examen ECAES-. Recogeré la experiencia de la Universidad Surcolombiana en la asunción que este comportamiento es común a todas las IES del país.

La Universidad Surcolombiana desde sus inicios -marzo de 1970- se ha caracterizado por intentar desarrollar una dinámica que responda a las necesidades del orden local, regional y nacional. Los diferentes planes de desarrollo institucional que se relacionan a continuación $y$, que sirven de marco de referencia para «contextualizar» el PEU (Acuerdo 020 de mayo de 2003), permiten afirmar que desde sus inicios la Universidad ha expresado su preocupación por responder a estas exigencias organizativas. Se puede afirmar que la preocupación por tener un norte definido ha sido una impronta que caracteriza a la USCO. 
La creación de ITUSCO (primer nombre que tuvo la Universidad Surcolombiana) hace parte de la política educativa que en la década del 70 del siglo anterior se impulsó con el propósito de ofrecer estudios y formación universitaria en algunos territorios y regiones consideradas deprimidas. Sin embargo, es necesario advertir que la presión de la comunidad, como también la fuerza y argumentos de las asociaciones de profesionales y del magisterio, lograron que efectivamente se decidiera la creación de una institución de educación superior para el Huila, de ahí, que en la memoria institucional sea frecuente encontrar crónicas relacionadas con la «institución que nació en la calle».

Tal como aparece en el PEU la Universidad nace con unos pocos programas $y$ posteriormente comienza a crecer en áreas del conocimiento relacionadas con salud, ingeniería, derecho, comunicación social y periodismo, economía y las ya existentes como eran educación y administración. La complejidad de este crecimiento exigió que se atendieran diversas necesidades relacionadas con los procesos de docencia, investigación y proyección social.

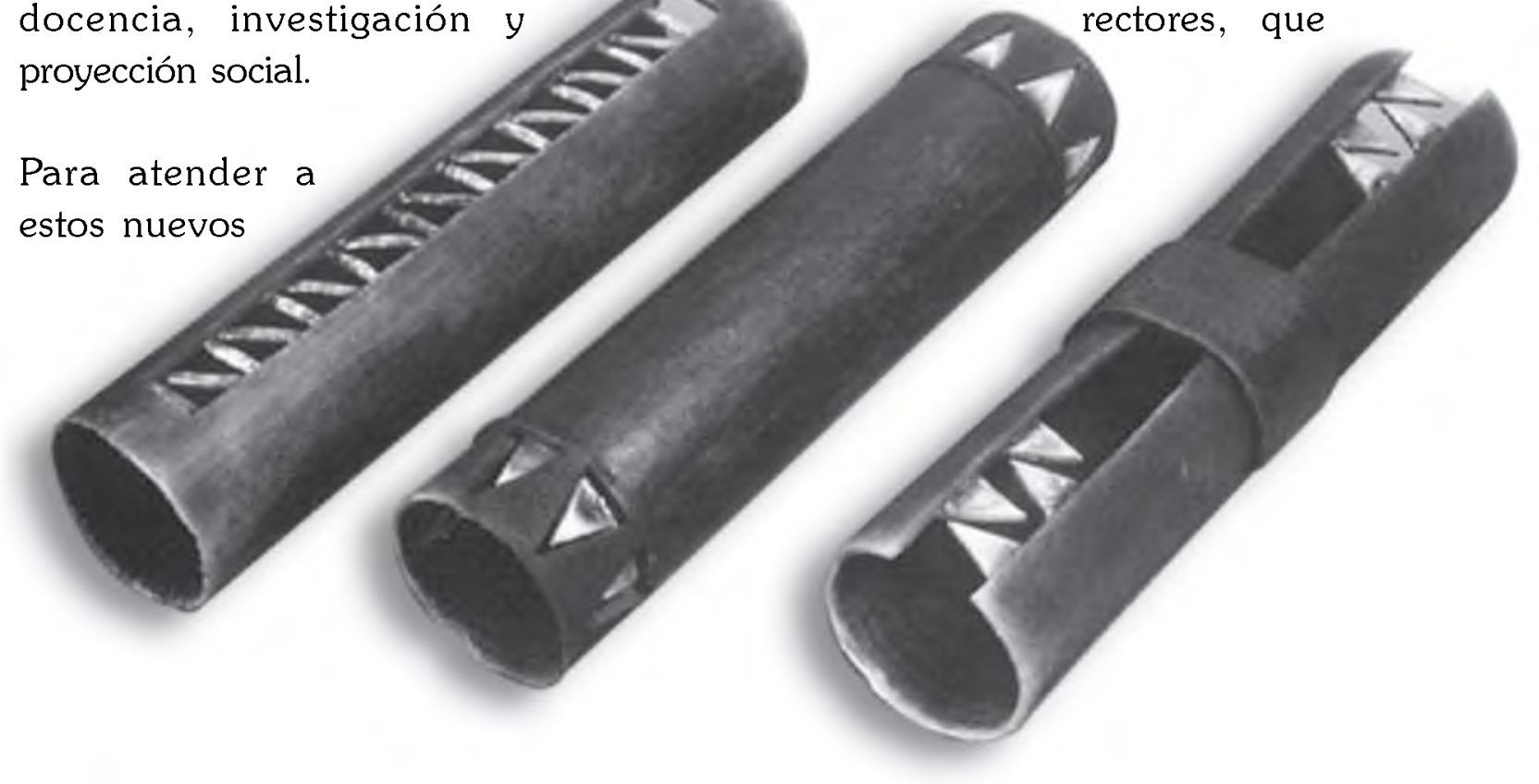

requerimientos la Universidad Surcolombiana consideró adecuado la formulación de planes de desarrollo que respondieran a las urgencias surgidas de este crecimiento, lo cual dio lugar a la elaboración y aprobación de los siguientes planes:
1. Plan de Desarrollo 1985 -1989
2. Plan de Desarrollo 1990-1994
3. Plan de Desarrollo 1997-2001
4. Plan de Desarrollo 2003-2007 ${ }^{9}$

La expedición del PEU se realiza en el contexto de ejecución del Plan de Desarrollo 2003-2007, lo cual permite advertir la complementariedad de estas dos disposiciones legales de carácter institucional. Además, es necesario consignar que la elaboración de estos dos elementos regulativos se da en el marco de las reivindicaciones de los estamentos de la comunidad educativa (docentes, estudiantes, investigadores, egresados, padres de familia, organizaciones sindicales, personal administrativo y de servicio) activadas por la crisis administrativa originada por la anulación de tres nombramientos de rectores, que 
originaron un caos y desorden institucional que de no ser por la existencia de un Discurso Pedagógico Institucional sólido (sustentado en políticas sobre docencia, investigación, proyección social, sistema docente, estudiantil, financiero, macroproyectos), perfectamente podrían haber originado el cierre o la intervención de la Universidad.

Se puede afirmar que el $\mathrm{PEU}^{10}$ en la actualidad se convierte en el referente obligado de la administración, es decir, no se desconoce que algunos de los elementos del PEU están en su etapa o fase de enunciación, no obstante el PEU es un factor determinante de su accionar. No se puede afirmar que todo lo que está consignado en el PEU se está desarrollando o ejecutando plenamente, sin embargo, lo que actualmente se ejecuta sí responde a lo consignado en el PEU.

La construcción, aprobación y puesta en práctica del PEU se realizó, sin que existiera la exigencia normativa y la obligatoriedad de participar en el ECAES (septiembre de 2003), razón por la cual, es conveniente ubicar en el plano hipotético la relación entre estas dos regulaciones que por su naturaleza son esencialmente diferentes, por un lado, el PEU se convierte en la carta de cartas de la Universidad; el ECAES, en la obligación derivada de la normatividad que configura el actual Discurso Pedagógico Oficial (Políticas Públicas); la una se desenvuelve en el marco de la autonomía universitaria, la otra, en el contexto de la heteronomía propiciada por el Estado. Por ello, es preciso indicar que el impacto del ECAES sobre la vida institucional, debe entenderse como una posible opción o referente a estudiar, no como una realidad dada o que deba darse.
El ECAES en la Universidad Surcolombiana se ha asumido como la obligación que tienen los estudiantes de último año de presentar dicho examen, como se puede colegir de la mesa de trabajo realizada que suministró información para la elaboración de este artículo, la percepción sobre el ECAES que tienen la mayoría de los miembros de la comunidad académica de la USCO no supera la dimensión del requisito a cumplir. La elaboración del PEU se puede caracterizar como el proceso mediante el cual, la mayoría de sus principios y propósitos están adquiriendo sentido de realidad. En la actualidad el PEU es un dispositivo de regulación de la administración de la Universidad, defendido y sostenido por la comunidad académica de la institución, se puede afirmar que no es algo coyuntural, por el contrario, responde a la naturaleza estructural de la Universidad.

Ahora bien, no se puede afirmar que no exista un vínculo entre el PEU y el ECAES, lo que es necesario aclarar y explicitar es que su relación no es de subordinación ni mucho menos de absorción. Es decir, no es posible determinar la calidad de un proceso formativo, ni la seriedad de la Universidad y del Programa a partir de los resultados del ECAES, esto sería otorgarle una condición y un carácter determinante a una experiencia que está en proceso de construcción y que además, solo es un factor. Qué tendrían que decir al respecto los procesos de Registro Calificado, de Acreditación de Calidad y el Observatorio Laboral. ¿no deben tenerse en cuenta estos otros factores para hablar de la calidad y excelencia de la Universidad y de sus procesos y agentes? 
Tampoco sería correcto ignorar la información suministrada por el ECAES, esta permite avanzar en procesos de reorientación y resignificación de las estructuras curriculares que sirven de base para la formación de profesionales en las diferentes áreas del conocimiento; de revisión sistemática de la naturaleza y el carácter de las prácticas pedagógicas que subyacen en el proceso de formación profesional; de la manera como se está organizando el conocimiento y los contextos de interacción que se activan en a través de las relaciones entre los agentes del proceso formativo. Innegable la importancia de la información del ECAES considerada objeto de investigación, es decir, es un medio pero no un fin en si mismo.

A partir de esta perspectiva resulta conveniente utilizar la información del ECAES como un pretexto para generar procesos y programas de investigación que permita, entre otros propósitos, avanzar en la construcción de respuestas satisfactorias frente a interrogantes como:

1. ¿Cuál es la pertenencia social y la pertinencia académica del actual portafolio académico de la USCO?

2. ¿ Qué se entiende por calidad y excelencia académica en la Educación Superior?

3. ¿ Cuál es la cultura evaluativa de la Universidad ¿Existe?

4. ¿Cuáles son las distancias y las cercanías entre los procesos de evaluación interna y externa que agencia la Universidad?

5. ¿Cuál es la voz que sobre la evaluación de la educación superior tiene la USCO?
Bien se podría hacer referencia a una gran cantidad de interrogantes sobre la problemática de la evaluación en la educación superior, sin embargo, considero que este artículo vería alcanzado su objetivo, si pudiera contribuir en la activación de un ambiente de análisis, reflexión y discusión sobre el fenómeno ECAES en la USCO, que propicie $y$ fomente un ambiente serio $y$ responsable de indagación sistemática y permanente que desborde la actitud maniquea y simplista de «especular» sobre los resultados de una prueba, que muy posiblemente es desconocida en su gramática básica por una gran cantidad de miembros de la comunidad académica.

Finalmente, considero oportuno invitar a la comunidad académica perteneciente a la educación superior en Colombia a que se «lea» la experiencia ECAES como un «reto» que debemos construir colectiva $y$ participativamente. Revisar la conveniencia de «instalar» en una sola institución (el ICFES) la legitimidad y la veracidad de este proceso. Si el universo de la educación superior en Colombia es rico y complejo en experiencias formativas, resulta paradójico que las IES no reaccionen de cara al interés implícito y explícito de segmentarlas y jerarquizarlas, como bien podemos observar a partir de la utilización pública de los resultados de la prueba ECAES.

El ECAES como experiencia académica es un proceso al cual no debemos otorgarle mayoría de edad o adultez, so pena de erosionar y debilitar la restringida autonomía universitaria que aun conservamos.

De que sirve contar con un Proyecto Educativo Universitario, con un Plan de 
Desarrollo concebido a varios años, con un Sistema Docente, Investigativo y de Proyección Social, con unas Políticas Institucionales en materia de procesos y procedimientos; si el comportamiento de un segmento mínimo de los estudiantes (último año), en un examen que está en proceso de validación, se convierte en la verdad irrefutable de calidad y de rigor formativo de la Universidad. Dejar pasar por alto estas reflexiones, es hacer uso de una licencia que posiblemente mañana generará oleadas de arrepentimiento.
Es necesario entender que todo proceso de evaluación debe ser asumido como una acción dialógica entre evaluador y evaluado. $\mathrm{Si}$ esto no se da estaremos en presencia de un acto de dominación entre "alguien» que cuenta con un poder y una gran cantidad de instituciones que solamente pueden hacer parte del "colonialismo académico» agenciado por ese «alguien»

Neiva, febrero 26 de 2006

\section{NOTAS}

(1) Ph.D. en Educación. Area Lenguaje y Educación. Director del Grupo de Investigación PACA-Programa de Acción Curricular Alternativo Categoría A Colciencia, Profesor Titular Facultad de Educación.

(2) Que la constitucionalidad del mismo esta en entredicho a partir de la Sentencia de la Corte C-852 se agosto de 2005

(3) Se pueden señalar como hitos históricos de la experiencia ECAES los siguientes:

1960 - Plan Nacional para la Educación Superior (Plan Básico); 1995 - Misión Nacional para la Modernización de la Universidad Pública; 2002 - Evaluación de 3 programas; 2003 - Evaluación de 27 programas; 2004 - Evaluación de 43 programas; 2005

- Evaluación de 52 programas.

(4) El marco legal de los ECAES está integrado por la Ley 30 de 1992, el Decreto 2566 de 2003 que establece condiciones mínimas de calidad; las resoluciones de características específicas de calidad de programas y el Decreto 1781 de 2003 reglamenta el ECAES.

(5) En las diferentes actividades desarrolladas por el ICFES es común encontrar esta afirmación

(6) Este término «competencia» ha sido objeto de las más variadas especulaciones y manejos dependiendo del contexto de su formulación. Interesante detenernos de manera rigurosa en el análisis de las implicaciones de esta concepción de cara a los «actuales» proceso de formación que adelantan las IES.

(7) En los actuales momentos a través de los diferentes medios de comunicación, se observa de manera sorprendente las diferentes declaraciones que funcionarios del MEN y del ICFES realizan en relación con los «resultados del ECAES 2005».

(8) Algunos recientes análisis sobre el ICFES señalan que hoy es una versión distorsionada 
de lo que fue el Servicio Nacional de Pruebas SNP, unidad que centró su accionar básicamente en el examen de Estado, es decir, en el examen que deben presentar los alumno(a)s de grado 11 . Su experiencia en la evaluación de los procesos de formación y calidad de la Educación Superior tiene de vida lo que tiene de vigencia el Decreto 1781/ 2003.

(9) En el Acuerdo 020 de 2003 por medio del cual se adopta el PEU se hace una descripción básica de cada uno de estos planes.

(10) Producto concreto del Comité de Autoevaluación y Acreditación conformado por tres representantes de cada facultad, un representante de los estudiantes, un representante de los egresados, dos representantes de los trabajadores y personal de servicios y coordinado por la Dirección General de Currículo adscrita a la Vicerectoría Académica en el año 2003. 